\title{
The 45th Annual Meeting of the European Society for Blood and Marrow Transplantation: Statistical Symposium - Poster Session
}

C Springer Nature Limited 2019

\section{4-27 March 2019 Frankfurt, Germany}

Modified and published with permission from https://www.ebmt.org/annual-meeting

Sponsorship Statement: Publication of this supplement is sponsored by the European Society for Blood and Marrow Transplantation. All content was reviewed and approved by the EBMT Committee, which held full responsibility for the abstract selections.

\section{P764}

A novel statistical approach for the comparison of conventional chemotherapy and stem cell transplantation in leukemia

\author{
Ulrike Pötschger ${ }^{1}$, Harald Heinzl$l^{2}$, Martina Mittlböck ${ }^{2}$ \\ ${ }^{1}$ Children's Cancer Research Institute (CCRI), S2IRP, \\ Vienna, Austria, ${ }^{2}$ Medical University of Vienna, Center for \\ Medical Statistics, Informatics, and Intelligent Systems, \\ Vienna, Austria
}

Background: Studies in the field of pediatric leukemia that aim to compare allogeneic stem-cell transplantation (SCT) with conventional chemotherapy are the motivation of this work. To avoid selection bias, the design of such studies is usually based on a so-called genetic randomization. This is a simple analysis based on donor availability status according to registered donors. However, as donor search is often ceased after a patient's event, donor availability status is incompletely observed, so that this simple comparison is not possible. Here, the waiting time to transplant, the anticipated non-proportional hazards (due to increased early toxicity and potentially decreased later disease recurrences with SCT) and the primary interest in long-term survival (i.e. cure) needs to be considered simultaneously. With nonproportional hazards, commonly used statistical methods like Cox-regression with time-dependent covariates and landmark analysis show several limitations (relying on the definition of landmark times, poor interpretability as hazard ratios instead of long-term survival probabilities are evaluated, low statistical power) and these limitations may lead to wrong conclusions. A novel statistical approach to overcome these shortcomings is presented here.

Methods: The pseudo-value regression technique is a powerful approach for modeling the impact of baseline covariates on long-term survival in a non-proportional hazards' situation. We generalized the original pseudo-value approach to allow an adjustment for waiting time to donor identification. Although donor availability is incompletely observed, our approach mimics a genetic randomization and unbiasedly estimates survival probabilities with and without a donor at pre-specified time-points.

The statistical evaluation is done in the framework of a statistical model. Hence, it is possible to adjust the analysis for potential risk-factors. This is a further major benefit of the given approach, that is useful in many practical applications. A further advantage of the proposed approach is the possibility to study the impact of waiting time on survival. Real data from childhood leukaemia are used to illustrate the practical value of the method. A simulation study was performed to investigate the statistical properties of the proposed model.

Results: Regardless of whether the proportional hazard assumption holds or not, the estimated parameters are unbiased. With non-proportional hazards our approach clearly outperforms commonly used methods, like Cox-regression with time-dependent covariates and landmark analysis, with respect to statistical power and interpretability of the results.

Conclusions: The proposed approach provides an unbiased, powerful and previously not available statistical tool to directly address the primary interest in survival probabilities in this common but methodologically difficult situation.

Clinical Trial Registry: None

Disclosure: Nothing to declare 


\section{P765}

Assessment of reporting quality of prediction model studies in HSCT: Adherence to the tripod statement

\section{Shuang Liu ${ }^{1,2,3}$, Weiluan Chen ${ }^{4}$, Pauline Heus ${ }^{5,6}$, Wenge Dong $^{1,2}$, Weihua Zhai ${ }^{1}$, Zhuang Cui ${ }^{3}$,Junfeng Wang ${ }^{7,8}$}

${ }^{1}$ Institute of Hematology and Blood Diseases Hospital, PUMC \& CAMS, Tianjin, China, ${ }^{2}$ Chinese Journal of Hematology, CMA, Tianjin, China, ${ }^{3}$ Tianjin Medical University, Tianjin, China, ${ }^{4}$ Utrecht University, Utrecht, Netherlands, ${ }^{5}$ Cochrane Netherlands, Utrecht, Netherlands, ${ }^{6}$ University Medical Center Utrecht, Utrecht, Netherlands, ${ }^{7}$ EBMT Leiden Data Office, Leiden, Netherlands, ${ }^{8}$ Leiden University Medical Center, Leiden, Netherlands

Background: We assessed the adherence to the TRIPOD statement of prediction model studies in the hematopoietic stem cell transplantation (HSCT) field, to evaluate the current reporting quality and possible future improvements.

Methods: We performed a comprehensive systematic literature search for prediction model studies in HSCT using MEDLINE and EMBASE from January 2000 through June 2018, complemented by looking at the references and consulting the experts. Candidate publications were assessed for eligibility using pre-defined inclusion criteria: (1) development and/or validation of prediction model/risk score; (2) outcome is an event after HSCT; (3) population is blood disease patients. Data was extracted from the final included studies on study characteristics (e.g. year of publication, journal of publication, authors information, etc.) and reporting quality which is evaluated using the Transparent Reporting of a multivariable prediction model for Individual Prognosis Or Diagnosis (TRIPOD) checklist. Adherence with each checklist item was examined, and the sum of adequately reported items was used to describe overall reporting quality. Publications were stratified to explore differences before and after the introduction of TRIPOD. The association between compliance and journal impact factor, involvement of statistician/epidemiologist, or length of publication was explored through univariate regression.

Results: A total of 9434 publications were identified by the search strategy, after removing duplicates, 7258 remained. After title and abstract screening, 336 publications were included for full-text check, and 154 publications were considered eligible for final analysis (Figure 1). They were heterogeneous in diseases, model types, and clinical outcomes. More than half $(53,9 \%)$ of the included studies are model validation. More models $(66,9 \%)$ were published before the launch of TRIPOD. Only in 63 studies (40,9\%), statisticians or methodologists were involved as coauthors
(Table 1). Compliance with TRIPOD was poor, especially in model validation studies.

Conclusions: Inadequate reporting can hamper the acceptance of the research results and the clinical application of prediction models. Authors, reviewers, and journal editors should all work on the completeness and accuracy of the reporting. TRIPOD can be applied to both model development and validation studies in HSCT. Encouraging use of TRIPOD might be helpful to further improvements in reporting quality.

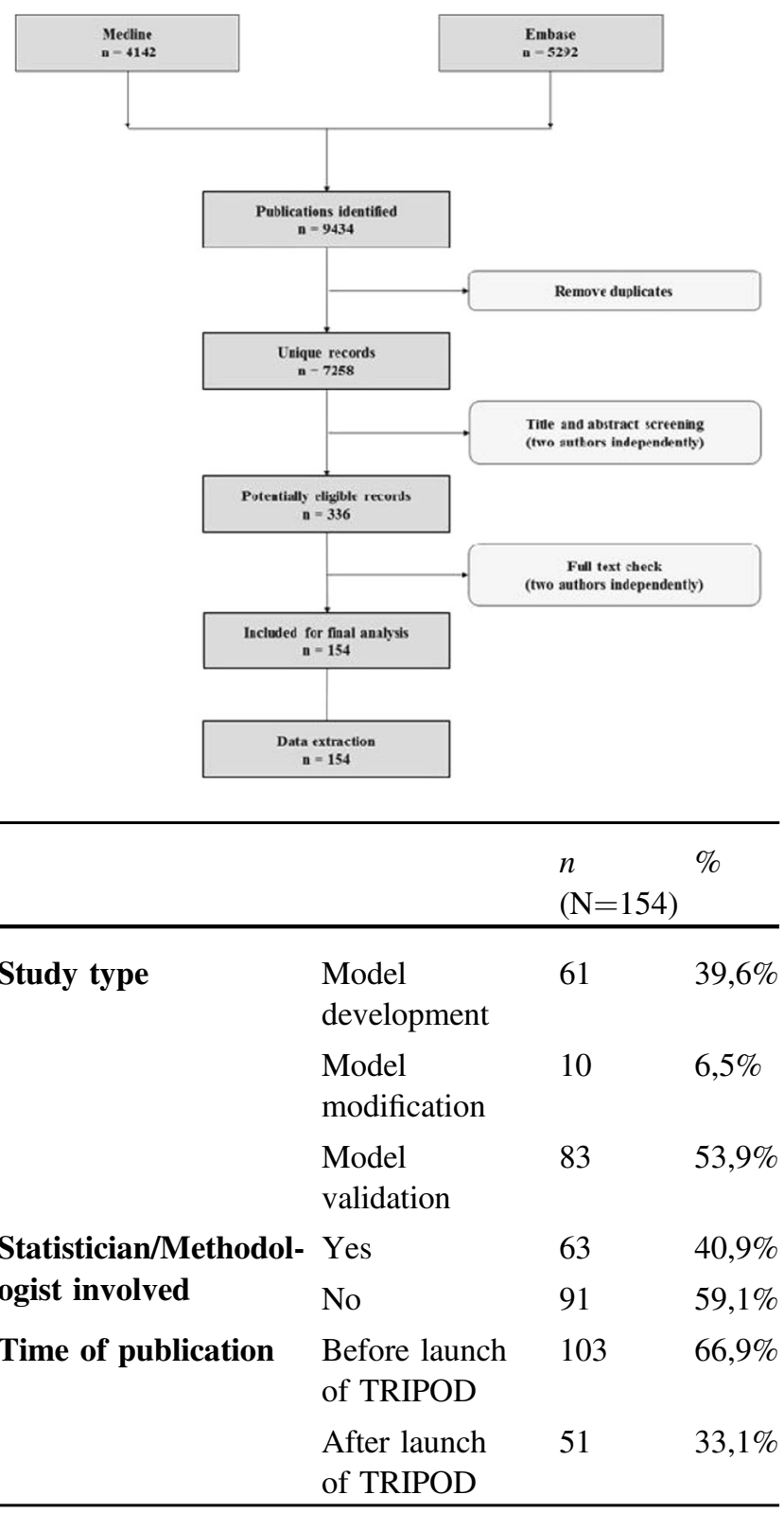

[[P765 Table] 1. Table 1. Basic information included publications]

Disclosure: Nothing to declare 